\title{
Mutual Interference in OFDM-based Spectrum Pooling Systems
}

\author{
Timo Weiss, Joerg Hillenbrand, Albert Krohn (IEEE Student Members), Friedrich K. Jondral (IEEE Senior Member)
}

\begin{abstract}
Public mobile radio spectrum has become a scarce resource while wide spectral ranges are only rarely used. Here, a new strategy called spectrum pooling is considered. It aims at enabling public access to these spectral ranges without sacrificing the transmission quality of the actual license owners. Unfortunately, using OFDM modulation in a spectrum pooling system has some drawbacks. There is an interaction between the licensed system and the OFDM based rental system due to the non-orthogonality of their respective transmit signals. In this paper, this interaction is described mathematically providing a quantitative evaluation of the mutual interference that leads to an SNR loss in both systems. However, this interference can be mitigated by windowing the OFDM signal in the time domain or by the adaptive deactivation of adjacent subcarriers providing flexible guard bands between licensed and rental system. It is obvious that both approaches sacrifice bandwidth of the rental system. A quantitative comparison of both approaches is given as a tradeoff between interference reduction and throughput in the rental system.
\end{abstract}

\section{INTRODUCTION}

The success of future wireless systems will depend on the concepts and technology innovations in architecture and in efficient utilization of spectral resources. There will be a substantial need for more bandwidth as wireless applications become more and more sophisticated. This need will not be satisfied by the existing frequency bands being allocated to public mobile radio even with very evolved and efficient transmission techniques. Measurement campaigns [1] have shown that wide ranges of potential spectral resources are used only very rarely. In the presented approach that is called spectrum pooling (SP), different spectrum owners (e.g. military, trunked radio etc.) bring their frequency bands into a common pool that rental users (RUs) may rent spectrum from. The notion of a spectrum pool was first mentioned in [2]. It reflects the need for a completely new way of radio resource management like in [3]. Interesting aspects of the spectral efficiency gain that is obtained with the deployment of SP are discussed in [4].

A potential rental system (RS) needs to be highly flexible with respect to the spectral shape of the transmitted signal. Here, the case of an FDMA/TDMA-based licensed system (LS) is considered. Thus, spectral ranges that are accessed by licensed users (LUs) have to be spared transmission power originating from the RUs. OFDM modulation is a candidate for such a system as it is possible to leave a set of subcarriers unused, thus providing a flexible spectral shape that fills the spectral gaps without interfering with the LUs. A detailed description of this approach is given in [5]. Furthermore, SP systems are not supposed to compete with existing and

T. Weiss, J. Hillenbrand, A. Krohn and F. K. Jondral are with the Institut fuer Nachrichtentechnik, Universitaet Karlsruhe, D-76128 Karlsruhe, Germany. E-mail: weiss@int.uni-karlsruhe.de upcoming $2 \mathrm{G}$ and $3 \mathrm{G}$ standards. They are rather meant to be a complement in hot spot areas with a high demand for bandwidth (e.g. airports, convention centers etc.). Hence, it is straightforward to apply modified versions of OFDM based wireless LAN standards like IEEE802.11a and HIPERLAN/2 [6], [7]. There are many modifications to consider in order to make wireless LANs capable of SP. They range from front end to higher layer issues [8]-[10].

This paper focuses on parasitic losses in SNR of both LS and RS due to the non-orthogonality of their respective transmit signals. A mathematical description of the disturbing impact of the OFDM based RS on the LS and vice versa is given in Sec. II and Sec. III, respectively. Sec. IV deals with possible countermeasures to the mutual interference of both systems and their impact on the system throughput of the RS.

\section{INTERFERENCE TO THE LICENSED SYSTEM}

This interference is caused by the side lobes of the OFDM signal. The transmit signal $s(t)$ on each single carrier of the considered wireless LAN standards [6], [7] is a rectangular NRZ signal. So, the power density spectrum (PDS) of $s(t)$ is represented in the form [11]

$$
\Phi_{s s}(f)=A^{2} T_{S}\left(\frac{\sin \pi f T_{S}}{\pi f T_{S}}\right)^{2},
$$

where $A$ denotes the signal amplitude and $T_{S}$ is the symbol duration which consists of the sum of useful symbol duration $T_{U}$ and guard interval $T_{G}$ [12]. It is assumed that the LS subbands are co-located with single subcarriers or sets of subcarriers [5]. First, the case is considered that the bandwidth of one LS subband is $\Delta f=1 / T_{U} . \Delta f$ represents the subcarrier spacing of the RS. The mean relative interference power to one LS subband $P_{R \rightarrow L}(n)$ is defined as

$$
P_{R \rightarrow L}(n)=\frac{1}{P_{t o t}} \int_{\left(n-\frac{1}{2}\right) \Delta f}^{\left(n+\frac{1}{2}\right) \Delta f} \Phi_{s s}(f) d f,
$$

where $P_{t o t}$ is the total transmit power emitted on one subcarrier and $n$ represents the distance between the considered subcarrier and the LS subband in multiples of $\Delta f$ which is illustrated in Fig. 1 for $n=1$. Calculating (2) with the parameters from the considered standards delivers row two of

\begin{tabular}{|c|cccc|}
\hline$n$ & 1 & 2 & 3 & 4 \\
\hline$P_{R \rightarrow L}(n)[\%]$ & 4.922 & 0.848 & 0.483 & 0.325 \\
\hline$P_{L \rightarrow R}(n)[\%]$ & 0.913 & 0.107 & 0.079 & 0.063 \\
\hline
\end{tabular}

TABLE I

RELATIVE INTERFERENCE POWER FROM RS TO LS AND VICE VERSA

table I. If the bandwidth of the LU subbands is a multiple of $\Delta f$, the total interference power of one subcarrier can be obtained by adding the values from this table. 


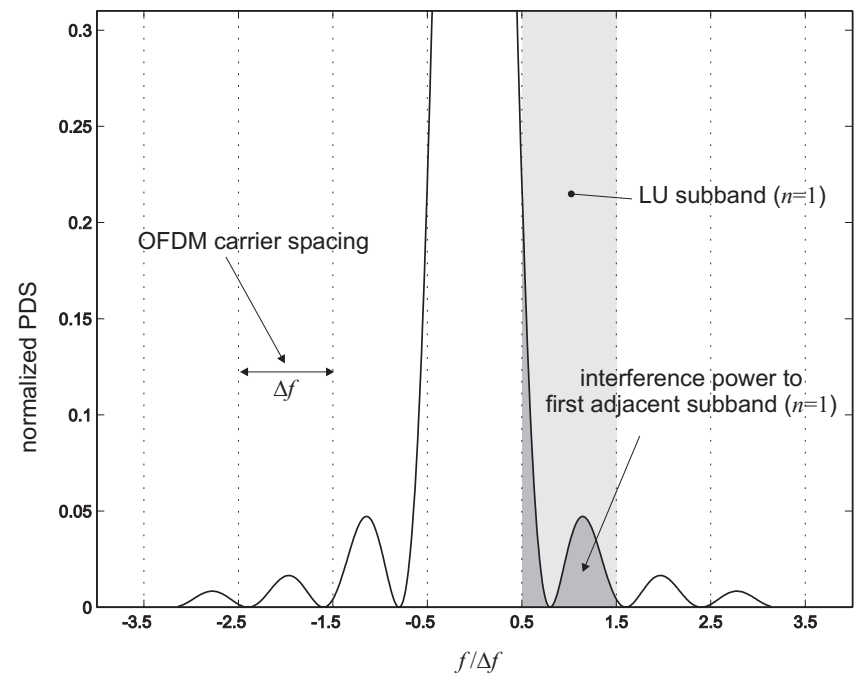

Fig. 1. PDS of a single OFDM modulated carrier in IEEE802.11a

\section{INTERFERENCE TO THE RENTAL SYSTEM}

The reception of an OFDM symbol is performed using an $N$-FFT function. This implies that the received signal $r(k)$ is windowed in the time domain by a rectangular window function $w(k)$ resulting in:

$$
\hat{r}(k)=r(k) w(k)
$$

Hence, the Fourier transform $\hat{X}\left(e^{j \omega}\right)$ of $\hat{r}(k)$ is represented by a convolution of the Fourier transforms $X\left(e^{j \omega}\right)$ and $W\left(e^{j \omega}\right)$ of their respective time signals $r(k)$ and $w(k)$. This yields:

$$
\hat{X}\left(e^{j \omega}\right)=\frac{1}{2 \pi} \int_{-\pi}^{\pi} X\left(e^{j \psi}\right) W\left(e^{j(\omega-\psi)}\right) d \psi
$$

If a rectangular window function is assumed, the PDS after the $N$-FFT processing can be expressed by the following expected value of the periodogram [13]

$$
E\left\{I_{N}(\omega)\right\}=\frac{1}{2 \pi N} \int_{-\pi}^{\pi} \Phi_{R R}\left(e^{j \omega}\right)\left(\frac{\sin (\omega-\psi) N / 2}{\sin (\omega-\psi) / 2}\right)^{2} d \psi,
$$

where $\omega$ represents the normalized frequency (normalized to the sampling frequency) and $\Phi_{R R}\left(e^{j \omega}\right)$ is the PDS of $r(k)$. It can be seen that the original spectrum $\Phi_{R R}\left(e^{j \omega}\right)$ is smeared by the convolution term in (5). This smearing does not destroy the orthogonality in a pure OFDM system but SP systems face a superposition with the LU signal. The impact of (5) on the LU signal is shown in Fig. 2. An elliptically filtered white noise process was assumed as LU signal. The circles indicate the sampling points of the 64-FFT in our example. One can see that significant parts of the LU energy are scattered to adjacent FFT bins where they interfere with the corresponding symbols of the OFDM transmission. Like in (2) the mean relative interference power to one subcarrier of the RS $P_{L \rightarrow R}(n)$ is defined. Taking the values from our example in Fig. 2 delivers the third row in table I. Although the effects between LS and RS and vice versa have a completely different physical and mathematical background, the order of magnitude of their

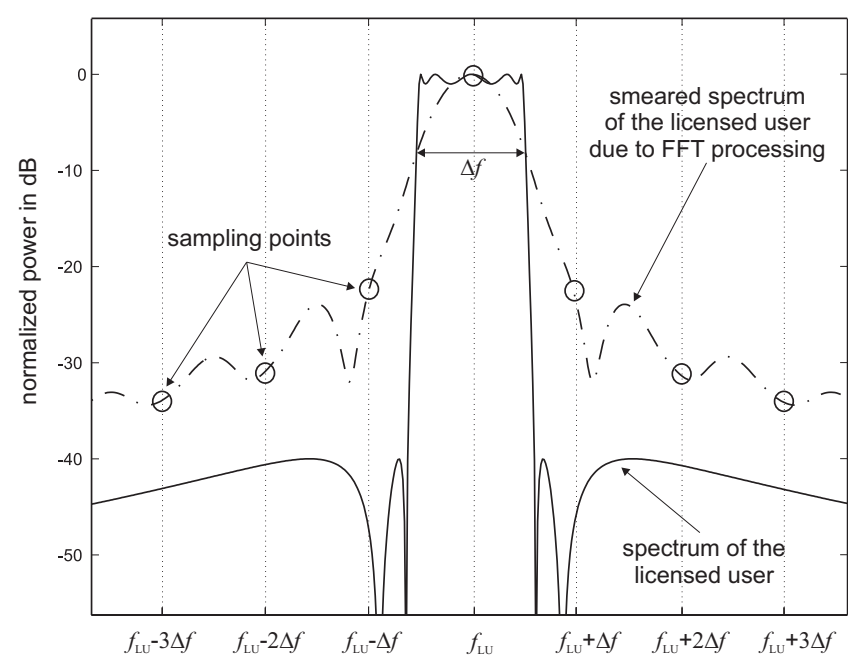

Fig. 2. Impact of the FFT processing on the PDS of the LU

interference impact is surprisingly similar as can be seen by comparing $P_{R \rightarrow L}(n)$ and $P_{L \rightarrow R}(n)$.

\section{Countermeasures to ThE MUtual INTERFERENCE}

One possible countermeasure to overcome the interference of the RS to LS is making the PDS in Fig. 1 go down more rapidly by windowing the transmit signal of the OFDM symbols. This makes the amplitude go smoothly to zero at the symbol boundaries. A commonly used window type is the raised cosine window that is defined by:

$g(t)= \begin{cases}\frac{1}{2}+\frac{1}{2} \cos \left(\pi+\frac{\pi t}{\beta T_{S}}\right), & \text { for } 0 \leq t<\beta T_{S} \\ 1, & \text { for } \beta T_{S} \leq t<T_{S} \\ \frac{1}{2}+\frac{1}{2} \cos \left(\frac{\pi\left(t-T_{S}\right)}{\beta T_{S}}\right), & \text { for } T_{S} \leq t<(1+\beta) T_{S},\end{cases}$

where $\beta$ denotes the rolloff factor. The symbol interval $T_{S}$ is shorter than the total symbol duration $(1+\beta) T_{S}$ because adjacent symbols are allowed to partially overlap in the rolloff region. The time structure of the OFDM signal using $g(t)$ as transmit filter is depicted in Fig. 3. It can be seen that the cyclic

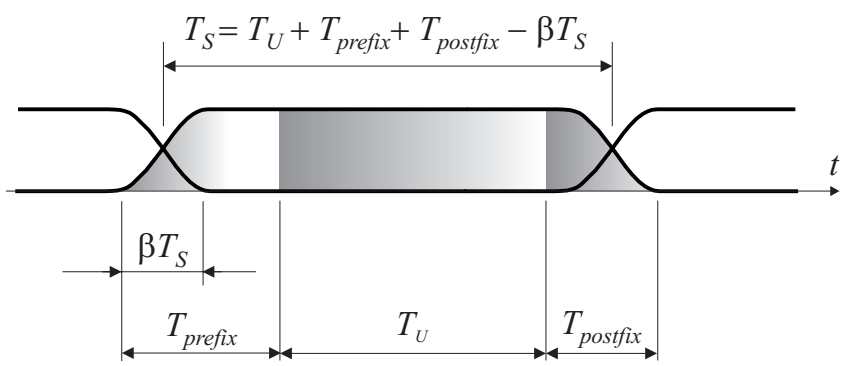

Fig. 3. Structure of the OFDM signal using a raised cosine transmit filter

prefix must be extended in order to achieve the same resistance against intersymbol interference (ISI). Furthermore, a cyclic postfix must be introduced. This postfix needs to be longer than $\beta T_{S}$ to maintain the orthogonality within the OFDM signal. Hence, the drawback of this interference reduction 


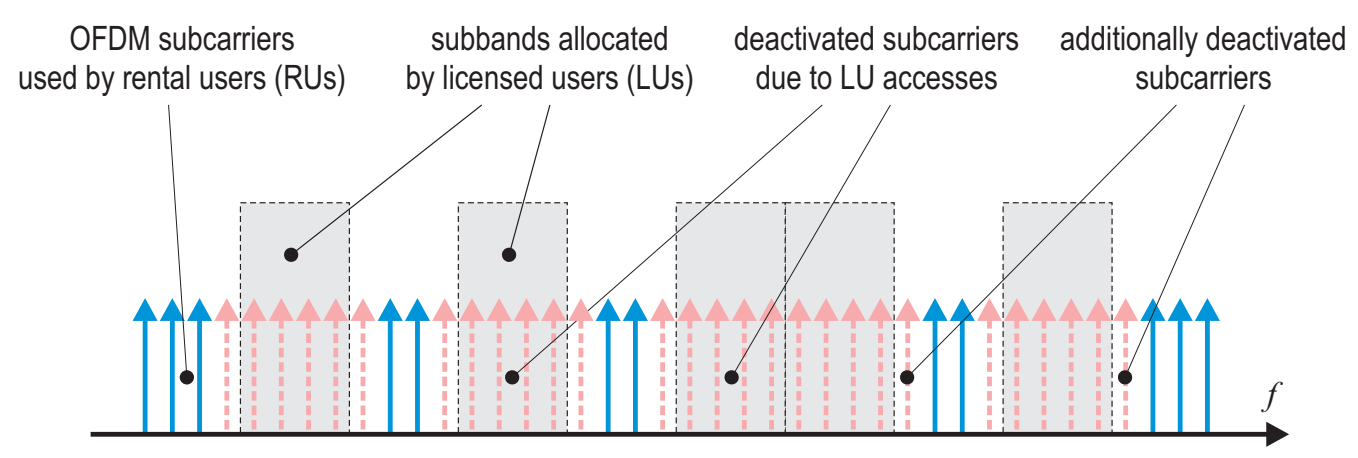

Fig. 6. Deactivation of adjacent subcarriers provides dynamic guard bands between LS and RS ( $b=1$ and $a=4$ in this example)

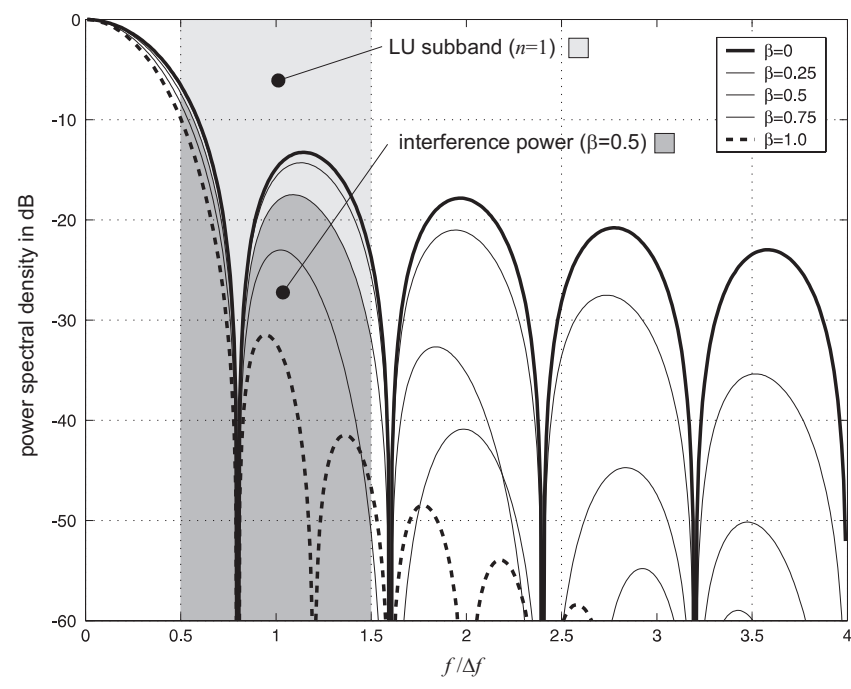

Fig. 4. Impact of the rolloff factor $\beta$ on the PSD of the rental user signal

method is the temporal extension of the symbol duration by the factor $(1+\beta)$ resulting in a reduced system throughput of the RS.

Fig. 4 shows the impact of the rolloff factor $\beta$ on the PDS of the RU signal. The side lobes are clearly attenuated. Again, it is assumed that the bandwidth of one LS subband matches one subcarrier spacing $\Delta f$. The first adjacent LS subband $(n=1)$ is illustrated in light gray. If the PDS is integrated like in (2), the resulting interference power (dark gray area) on the individual LS subband can be calculated.

The results of this calculation depending on the rolloff factor and the number of the LS subband is shown in Fig. 5. The positive effect of the raised cosine filter is stronger regarding LS subbands that are farther away from the considered RS subcarrier $(n \geq 2)$. Unfortunately, the benefit of the raised cosine filter is very small for the first adjacent subband, which can be seen from the almost constant curve for $n=1$. Even at very high rolloff factors, the achievable interference reduction is only about $6 \mathrm{~dB}$.

Therefore, the method of raised cosine filtering can only be considered an additional means to reduce the interference in the LS, but more powerful techniques need to be developed. One further interference reduction concept, which is presented here, is the dynamic deactivation of subcarriers lying

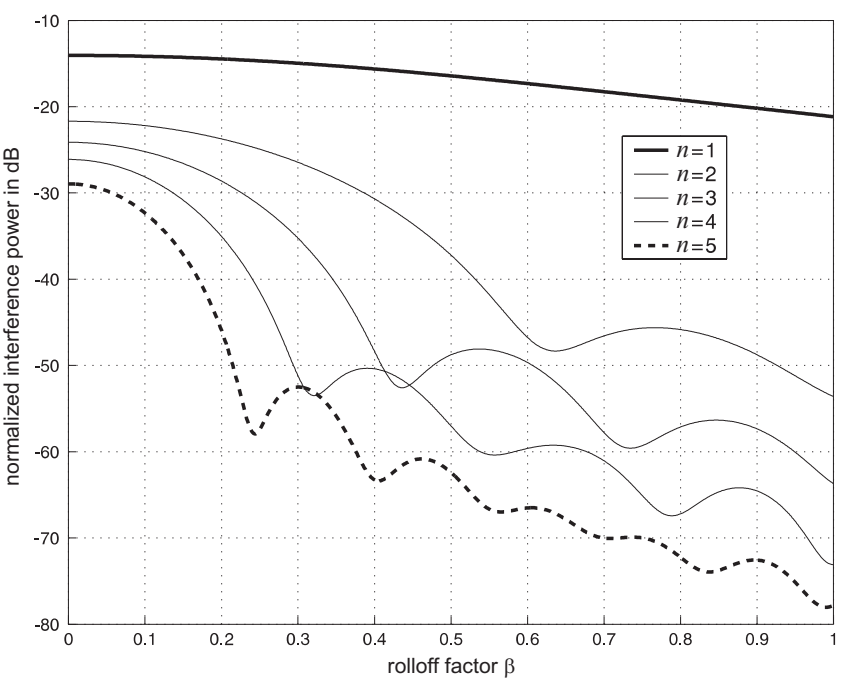

Fig. 5. Interference power in different LU subbands as a function of $\beta$

adjacently to allocated LS subbands. This provides flexible guardbands as pointed out in Fig. 6. The number of subcarriers that is covered by one LS subband is denoted by $a$ while the number of deactivated adjacent subcarriers is described by $b$. The advantage of this deactivation procedure compared to the raised cosine method is that both types of interference can be mitigated at once. Again, this sacrifices bandwidth and system throughput in the RS. A tradeoff needs to be found between tolerable disturbance to the LS and the remaining bandwidth of the RS.

In this paper we focus on the reduction of interference with the LS as this is the dominant interference effect (see table I). In order to obtain the mean interference power to one LS subband $P_{R \rightarrow L}$, all interference contributions of every single RS subcarrier need to be cumulated. Of course, wider LS subbands face more interference power as the integration width is larger. Hence, $P_{R \rightarrow L}$ is normalized to the corresponding bandwidth $a$ of one LS subband counted in integer multiples of $\Delta f$ :

$$
\bar{P}_{R \rightarrow L}=\frac{1}{a} P_{R \rightarrow L}
$$

Hence, $\bar{P}_{R \rightarrow L}$ can be interpreted as a mean relative interference power "density" that is caused by the RS. Another 
important parameter is the relative pool occupancy $r$ that represents the number of subcarriers that is occupied by the access of the LUs relative to the maximum number of available subcarriers. Of course, the interference power in one LS subband depends on the LU constellation within the considered pool. For this reason all possible constellations were simulated to obtain $\bar{P}_{R \rightarrow L}$ with varying parameters $b$, $a$, and $r$. One example of the simulation results is given in

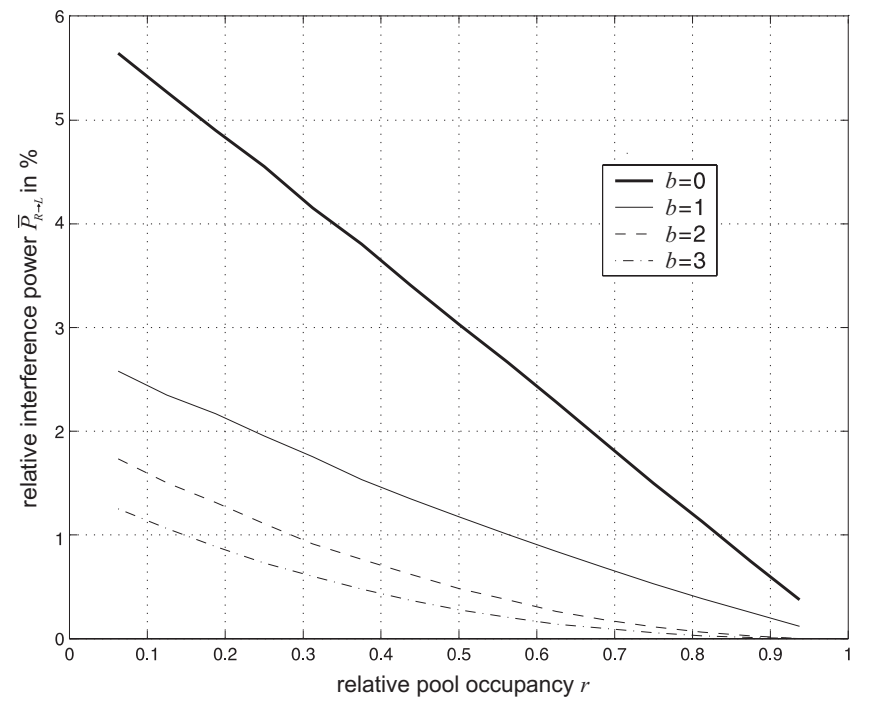

Fig. 7. Impact of the number of deactivated adjacent subcarriers $b$ on $\bar{P}_{R \rightarrow L}$

Fig. 7. It can be seen that $\bar{P}_{R \rightarrow L}$ is monotonically decreasing with increasing occupancy $r$. This is due to the fact that there are fewer interfering RS subcarriers left if more LS

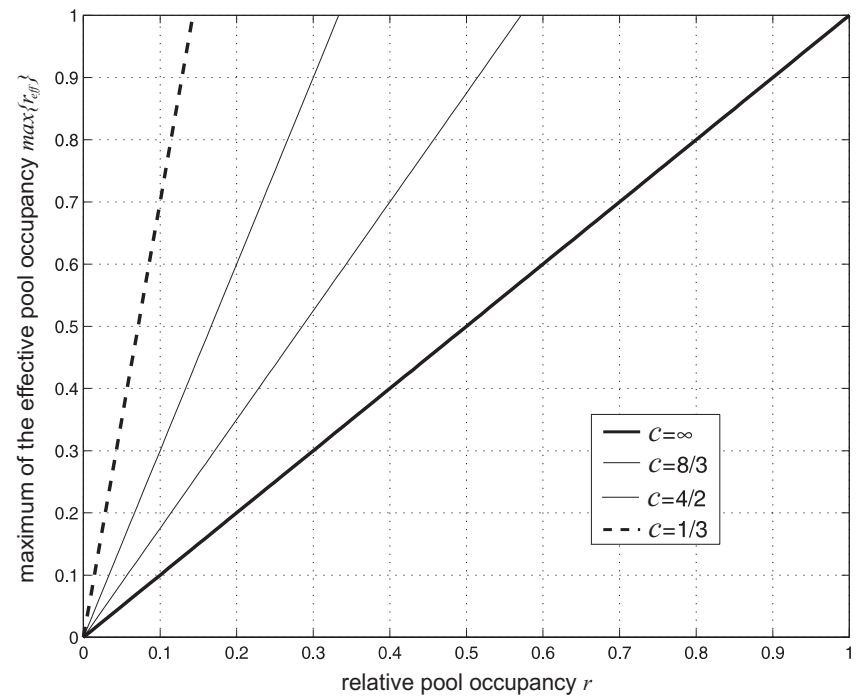

Fig. 8. $\max \left\{r_{\text {eff }}\right\}$ as a function of the relative pool occupancy $r$

subbands are occupied. Another result is that the deactivation of the first adjacent subcarrier $(b=1)$ delivers the largest benefit. The additional deactivation of more subcarriers $(b \geq 2)$ only provides a minor further improvement. A result of these simulations, which is not shown here, is that a larger access width $a$ is advantageous when it comes to the reduction of $\bar{P}_{R \rightarrow L}$. An explanation is that there are less split spectral ranges that interfering subcarriers can jump into.

It is straight forward that the reduction of interference and the highest possible throughput are contradicting requirements. Hence, a tradeoff is required. After the quantitative evaluation of the interference reduction by deactivating subcarriers as presented above, the impact of this method on the throughput of the RS needs to be investigated. First, the effective relative pool occupancy $r_{\text {eff }}$ is introduced representing the number of occupied subcarriers including the additionally deactivated subcarriers. If no subcarriers are deactivated $r$ and $r_{\text {eff }}$ are identical. Of course, $r_{\text {eff }}$ depends on the constellation of the pool. A worst case consideration regarding the constellation is that there are no neighboring LU subbands in the pool. In this case $2 b$ subcarriers are deactivated for each LU subband ( $b$ for each side). Thus, the maximum effective relative pool occupancy can be described by:

$$
\max \left\{r_{\text {eff }}\right\}= \begin{cases}\left(\frac{2 b}{a}+1\right) r, & \text { for } 0 \leq r<\frac{a}{a+2 b} \\ 1, & \text { for } \frac{a}{a+2 b} \leq r \leq 1\end{cases}
$$

The introduction of the substitution $c=\frac{a}{b}$ yields:

$$
\max \left\{r_{\text {eff }}\right\}= \begin{cases}\left(\frac{2}{c}+1\right) r, & \text { for } \quad 0 \leq r<\frac{c}{c+2} \\ 1, & \text { for } \quad \frac{c}{c+2} \leq r \leq 1\end{cases}
$$

The graph of this function is visualized in Fig. 8. In order to derive an analytical solution for $E\left\{r_{e f f}\right\}$ one has to take a closer look at the probabilities of sequences of occupied LS subbands in the allocation vector [10]. In the following, an occupied LS subband is represented by $a$ ones while an idle LS subband is described by $a$ zeros. The allocation vector is assumed to be large enough so that the margins do not need a separate consideration. For the sake of simplicity, $a=1$ is assumed. The probability that a sequence of $k$ consecutive occupied LS subbands occurs in the allocation vector is

$$
P(\underbrace{11 \ldots 1}_{k \text { times }} 0)=r^{k}(1-r), \quad \text { for } \quad \mathrm{k} \geq 1
$$

If $b$ adjacent subcarriers are deactivated with an LU access width of $a$ subcarriers and a sequence length of $k$, the local effective relative pool occupancy is

$$
r_{\text {eff }}=\frac{k a+2 b}{(k+1) a}=\frac{k+\frac{2}{c}}{k+1}
$$

Hence, the expected value $E\left\{r_{\text {eff }}\right\}$ can be expressed by [14]:

$$
\begin{aligned}
& E\left\{r_{\text {eff }}\right\}=(1-r) \sum_{k=1}^{\infty} \frac{k+\frac{2}{c}}{k+1} \cdot r^{k} \\
& = \begin{cases}\left(\frac{2}{c}+1\right) r-\frac{2}{c} r^{2}, & \text { for } \quad \mathrm{c} \geq 2 \\
\left(\frac{2}{c}+1\right) r-\left(\frac{4}{c}-1\right) r^{2} & \\
+\left(\frac{2}{c}-1\right) r^{3}, & \text { for } \quad 1 \leq \mathrm{c}<2\end{cases}
\end{aligned}
$$

This result is visualized in Fig. 9. The curves for $c<1$ were generated by simulation. It can be seen that only few spectral resources remain in the RS if the parameter $c$ gets large. Therefore, the number of deactivated subcarriers $b$ must be as small as possible. A tradeoff can be found if one allows 


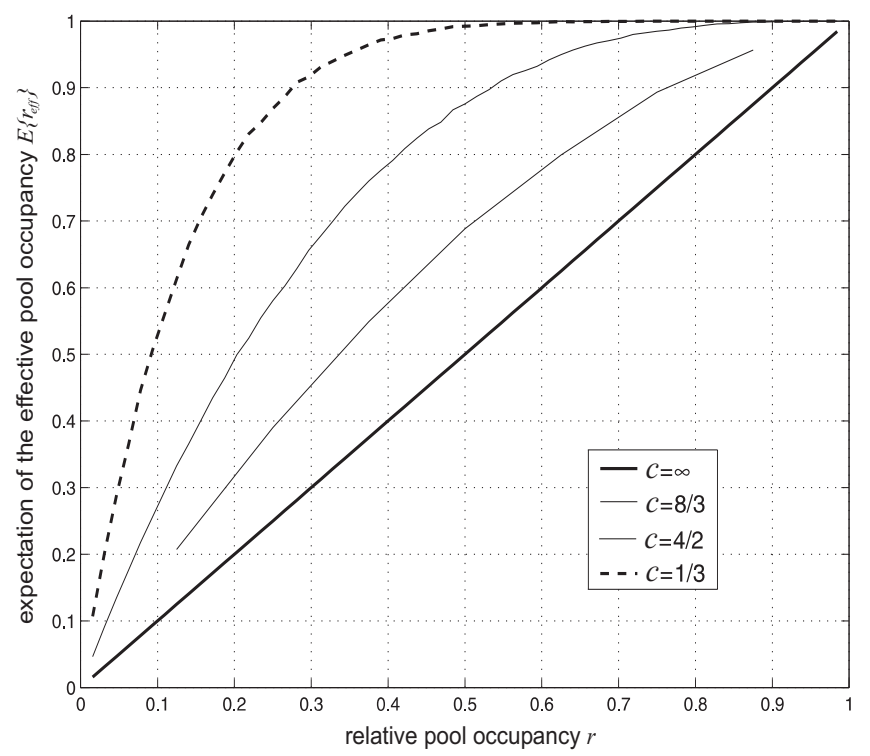

Fig. 9. $E\left\{r_{\text {eff }}\right\}$ as a function of the relative pool occupancy $r$

of certain interference level in LS, e.g, $\bar{P}_{R \rightarrow L}=2 \%$ like in Fig. 10. Then, the lowest possible $b$ has to be applied to guarantee the interference requirements. If the pool occupancy rises, fewer subcarriers need to be deactivated still observing the interference rule. This adaptive deactivation provides the highest possible throughput in the RS and ensures that a given interference threshold is not exceeded. The switching points for $b$ are shown in Fig. 10.

\section{CONCLUSION}

OFDM is a promising candidate for SP as it allows a very flexible radio resource management on a carrier-by-carrier basis. However, it has been shown that using OFDM has the drawback of mutual interference between RS and LS. A quantitative evaluation of the interference caused by the RS has been given. The interference from the LS occurs in the OFDM receivers of the RS due to the rectangular windowing of the receive signal in the time domain. Simulation results for this type of interference have been given. We proposed two possible countermeasures in order to mitigate the mutual interference. The first one is based on raised cosine windowing techniques for the OFDM signal. Analysis has shown that the benefit of this approach with respect to interference reduction is fairly low. A more powerful method has been proposed based on the dynamic deactivation of subcarriers providing flexible guard bands between LS and RS. The performance of this approach has been validated by simulation. Furthermore, a tradeoff between interference reduction and possible throughput has been presented.

\section{REFERENCES}

[1] F. Capar, T. Weiss, I. Martoyo, and F. Jondral, "Analysis of Coexistence Strategies for Cellular and Wireless Local Area Networks," Vehicular Technology Conference, 2003. VTC Fall 2003, Orlando, FL USA, October 2003.

[2] J. Mitola, "Cognitive Radio for Flexible Mobile Multimedia Communications," IEEE Int. Workshop on Mobile Multimedia Communications, San Diego, CA USA, November 1999.

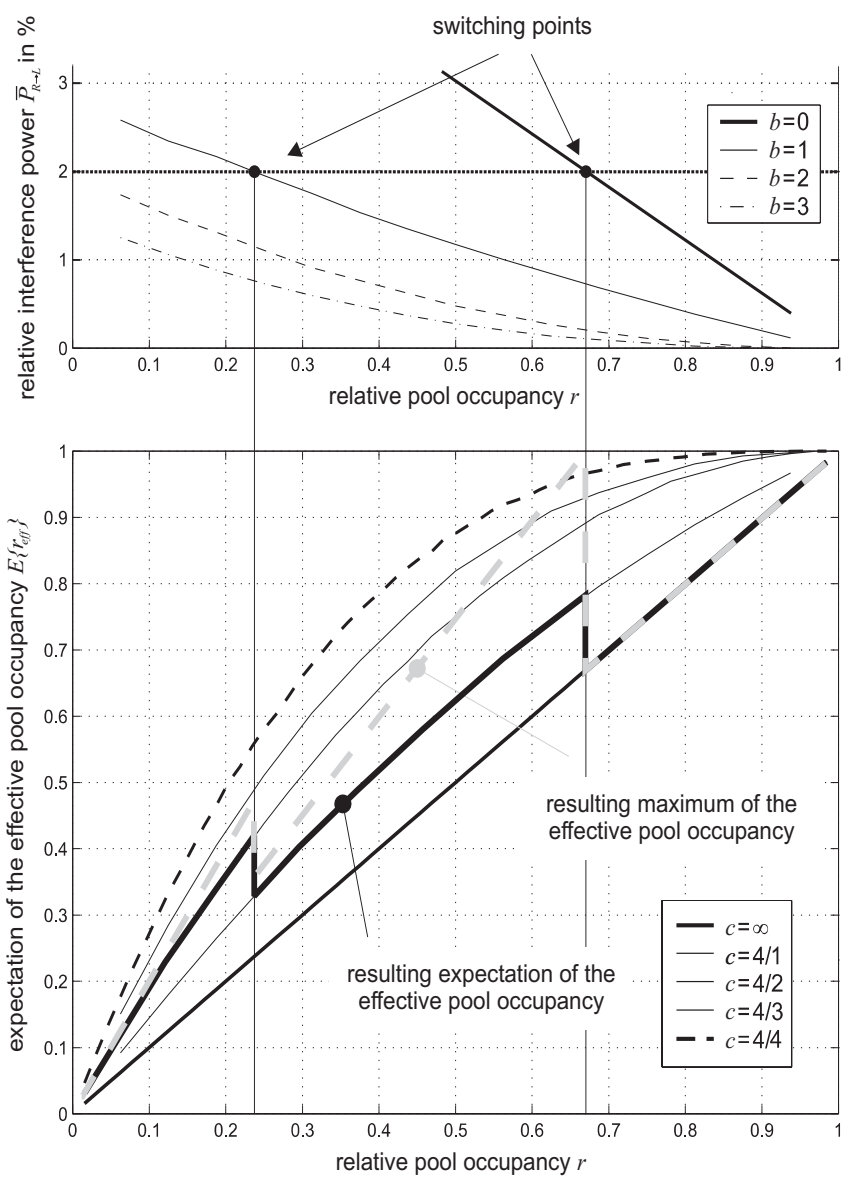

Fig. 10. Dynamic guard bands depending on the relative pool occupancy $r$

[3] E. Noam, "Taking the Next Step Beyond Spectrum Auctions: Open Spectrum Access," IEEE Communications Magazine, vol. 33, no. 12, December 1995.

[4] F. Capar, T. Weiss, I. Martoyo, and F. Jondral, "Comparison of Bandwidth Utilization for Controlled and Uncontrolled Channel Assignment in a Spectrum Pooling System," Vehicular Technology Conference, 2002. VTC Spring 2002, Birmingham, AL USA, March 2002.

[5] T. Weiss and F. Jondral, "Spectrum Pooling - An Innovative Strategy for the Enhancement of Spectrum Efficiency," IEEE Communications Magazine, vol. 42, no. 3, March 2004.

[6] IEEE802.11a, "Wireless LAN Medium Access Control (MAC) and Physical Layer (PHY) Specifications: High-speed Physical Layer in the 5 GHz Band," IEEE, Tech. Rep., 1999.

[7] ETSI-TS-101475, "Broadband Radio Access Networks (BRAN); HIPERLAN Type 2; Physical (PHY) Layer," ETSI, Tech. Rep., 2001.

[8] T. Weiss, A. Krohn, and F. Jondral, "Synchronization Algorithms and Preamble Concepts in Spectrum Pooling Systems," IST Mobile and Wireless Telecommunications Summit, Aveiro, Portugal, June 2003.

[9] T. Weiss, J. Hillenbrand, and F. Jondral, "A Diversity Approach for the Detection of Idle Spectral Resources in Spectrum Pooling Systems," Proc. of the 48th Int. Scientific Colloquium, Ilmenau, Germany, September 2003.

[10] T. Weiss, J. Hillenbrand, A. Krohn, and F. Jondral, "Efficient Signaling of Spectral Resources in Spectrum Pooling Systems," Proc. of the 10th Symposium on Communications and Vehicular Technology SCVT 2003, Eindhoven, Netherlands, November 2003.

[11] J. Proakis, Digital Communications. McGraw-Hill, 2001.

[12] R. van Nee, OFDM for Wireless Multimedia Communications. Artech House Publishers, 2000.

[13] A. Oppenheim and R. Schafer, Digital Signal Processing. Prentice-Hall, 1975.

[14] I. M. Ryshik and I. S. Gradstein, Tables of Series, Products, and Integrals. VEB Deutscher Verlag der Wissenschaften, 1963. 